\title{
Development of active agent (essential oil) releasing automotive interior head-inner using pet fabric
}

\begin{abstract}
Polyester fabric finds its extensive use in the automobile soft trim, in forms of seating trims, head liners and car panels. In this research, a 100\% PET fabric which can find its use as a headliner is subjected to surface treatment such that it can be a sustainable active agent (fragrance) releasing system and help in improving the interior ambience of the automobile. For a $100 \%$ polyester fabric, it's difficult to attach new functional groups on its surface because of its chemical nature. To overcome this problem, surface modification of polyester is required such that it has enough available hydroxyl (OH-) groups and carboxylate (R-COO-) groups which would aid in the attachment of the active agent releasing finish on the polyester. The active agent releasing complex used here is $\beta$-Cyclodextrin, which due to its toroidal shape and lipophilic cavity is capable of entrapping the lipophilic essential oil molecule and help in its controlled release over a longer period of time.
\end{abstract}

Volume 2 Issue 5 - 2017

\author{
Roopkatha Pallye,' Subhas Ghosh,' Debasish \\ Das $^{2}$ \\ 'College of Technology, Eastern Michigan University, USA \\ ${ }^{2}$ Department of Jute and Fiber Technology, University of \\ Calcutta, India
}

\begin{abstract}
Correspondence: Subhas Ghosh, College of Technology, Eastern Michigan University, Michigan, USA, Tel 7344872476, Fax
\end{abstract} 7344877087,Email sghosh@emich.edu

Received: July 07, 2017 | Published: August 16, 2017

\section{Introduction}

In the emerging trends of the textile industry, the incorporation of cosmetics/medicine/aroma in textile fabrics has been expanded with the development of sustainable time- release active agents such agents as perfume, odor-trapping components, medicines, antioxidants, moisturizer and regenerators. This study develops an activeagent releasing finish on cellulosic fabric and cellulosic blend fabric. Aromatherapy is the use of concentrated essential oils extracted from herbs, flowers and other plants to treat various types of ailments. Brown ${ }^{1}$ reported the effects of individual fragrances on human health. Essential oils, like all organic compounds, are made up of complex mixtures of several hydrocarbons such as terpenes, alcohols, esters, aldehydes, ketones, oxides and phenols. Even a simple essential oil may contain 80-300 different chemical constituents as reported by $\mathrm{Hu}$ et $\mathrm{al}^{2}{ }^{2}$ Authors described the creation of a perfume release fabric using rose fragrance nano-capsules directly on finished cotton fabrics. The authors claimed an excellent sustained fragrance release property. Wang \& $\mathrm{Chen}^{3}$ used aromatherapy oil on textile fabric after treating the fabric with $\beta$-Cyclodextrin, where the inclusion compound was applied to the fabric using the traditional pad method. Essential oil has been used alone or along with Western-style medical treatment in many different cultures. ${ }^{4}$ Perfume releasing cotton fabric has been developed by microencapsulation or grafting techniques. The second one is more economic and environment friendly. The cotton fiber is composed of $98 \%$ cellulose. In the grafting technique, cellulose chains of cotton containing free hydroxyl groups are attached to an inclusion complex of $\beta$-Cyclodextrin which releases the fragrance from the fabric in a time release basis. In automotive interior trim polyester fabric is used owing to its strength, less cost and durability under extreme weather conditions. The $\beta$-Cyclodextrin is a cyclic oligosaccharide compound of six glucose units linked by $\alpha-1,4$ glycoside bonds. They are reported to be produced by the enzymatic degradation of starch by means of $\alpha$-glucosidase or $\alpha$-amylase. ${ }^{5}$ It has a conical conformation with a height approximately $0.78 \mathrm{~nm}$ and diameter 0.57 to $0.95 \mathrm{~nm}$. Each Cyclodextrin has a hydrophobic cavity which acts as a host for the aromatherapy essential oils as shown in Figure 1. This cavity that can host the $\beta$-Cyclodextrin is mostly used on textiles because of ease of production, price and size of the cavity. All glucopyranose units in the torus-like ring are reported to possess the thermodynamically favored chair conformation because all substituent are in the equatorial position. Hydroxyl groups on the outside of the Cyclodextrin ensure good water solubility. However, the cavity of the ring is lined with hydrogen atoms of $\mathrm{C} 3$, by the glycoside oxygen bridges and hydrogen atoms of $\mathrm{C} 5$ making the inner side of such ring appreciably hydrophobic. The $\mathrm{C}-2-\mathrm{OH}$ group of one glucopyranoside unit can form a hydrogen bond with the $\mathrm{C}-3-\mathrm{OH}$ group of the adjacent glucopyranose unit. Cyclodextrin behaves more or less like rigid compounds. The Cyclodextrin complex formation is a dimensional fit between the host cavity and guest molecule (essential oil). The lipophilic cavity of Cyclodextrin molecule provides a microenvironment into which appropriately sized non-polar moieties can enter to form inclusion complexes. No covalent bonds are broken or formed during the formation of the inclusion complex. The driving force of a complex formation is the release of enthalpy-rich liquid (water) molecules from the cavity. The binding strength depends on how well the host-guest complex fits together and on specific local interactions between surface atoms. Complexes can be formed either in solution or in the crystalline state and a suitable solvent including water.

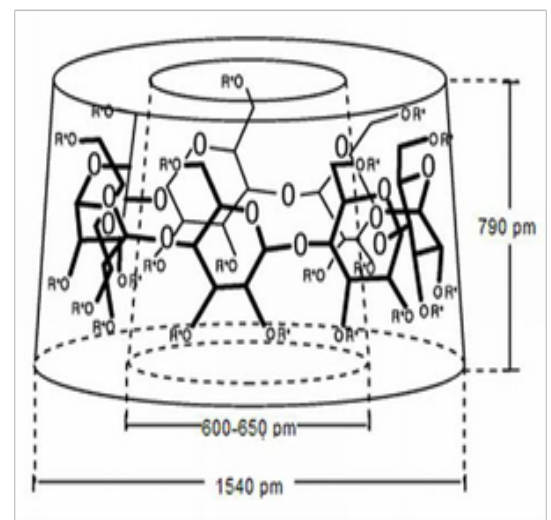

Figure I Schematic Structure of $\beta$-Cyclodextrin. 
Polyester fabric contains polyethylene terephthalate (PET) chains which has only few hydroxyl end groups that are not enough to be attached to $\beta$-Cyclodextrin. Therefore, in order to develop a chemically modified active agent releasing polyethylene terephthalate (PET) polyester fabric we partially de-esterified to generate sufficient amount of free $\mathrm{COOH}$ groups on the surface. This de-esterification has been carried out under control conditions so that the process does not degrade the fabric or any other properties like color, stiffness, appearance.

\section{Method and materials}

\section{Material}

A 100\% Polyester (PET) knitted fabric normally used in automotive head liners was used. Surface Modification (de-esterification) of Polyester through hydrolysis in alkali medium. The de-esterification of Polyester was carried out using $2 \%$ sodium hydroxide $(\mathrm{NaOH})$ solution, the chosen alkaline medium, in the presence of $1 \%$ accelerator (Titanium Butoxide), and the temperature and duration of hydrolysis was adjusted for optimum de-esterification. The samples were dried at $60^{\circ} \mathrm{c}$ and then they were washed with soap and further dried in ambient condition. $\beta$-Cyclodextrin was obtained from Sigma Aldrich and used as received. All other chemicals were also purchased from Sigma Aldrich and used without further purification.

\section{Re-esterification with active agent releasing agent ( $\beta$-Cyclodextrin)}

$2 \%, 4 \%$ and $6 \% \beta$-Cyclodextrin were administered separately by Pad-dry-cure method into the de-esterified samples. Citric acid (a carboxylic acid) was used as a cross linking agent between the $\beta$-Cyclodextrin and generated end groups in polyester chains in presence of a catalyst di sodium hydrogen phosphate, both mixed into the solution at the same percentage as Beta-Cyclodextrin. The fabric was soaked in the solution for 10minutes and then padded under pressure at padding mangles with 4dip-4nip. Then it was left to dry at room temperature.

\section{Application of fragrance}

$50 \%$ by volume of eucalyptus essential oil dissolved in methylated ethanol was force- sprayed on the de-esterified, beta-Cyclodextrin treated samples and left to dry at room temperature. A schematic of application is shown in Figure 2.

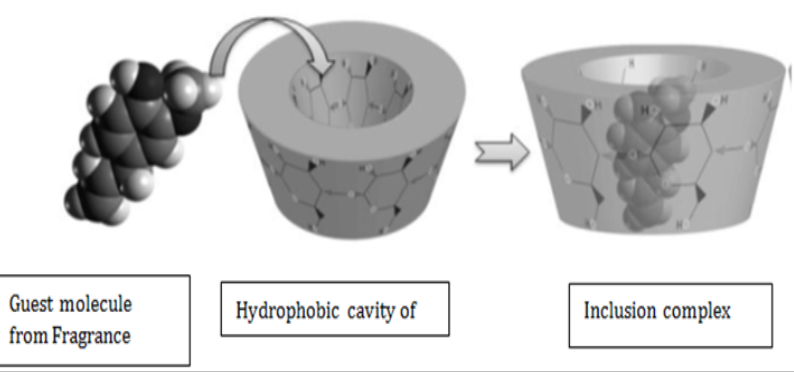

Figure 2 Formation of Inclusion Complex.

\section{Results and discussion}

\section{Fourier transformed infrared (FTIR) spectroscopy analysis}

Polyester fabrics used in this investigation were analyzed before and after de-esterification by a Bruker instrument, model: Tensor 27 spectrophotometer. $1780-1710 \mathrm{~cm}^{-1}$ peaks belong to $\mathrm{C}=\mathrm{O}$ stretching vibration arising from the carboxylic acid group in PET.As seen in Figure 3 the increase in the intensity of the peak at wavelengths 1714 and $1712 \mathrm{~cm}^{-1}$ confirmed the effect of hydrolysis due to the deesterification of the polyester fabric.

Figure 4 shows the FTIR spectra of $\beta$-Cyclodextrin and deesterified polyester. A small band between $3200-3600 \mathrm{~cm}^{-1}$ can be seen on the de-esterified neat PET spectra associated $\mathrm{OH}$ stretch in the ester group region; however, the intensity of the band greatly increased as seen in the spectra of the CDs treated PET fabric which is due to the presence of OH stretch vibrations at $3200-3600 \mathrm{~cm}^{-1}$ of $\beta$-Cyclodextrin molecules. Figure 5 illustrates the increase in peak intensity at 3200$3600 \mathrm{~cm}^{-1}$ as the concentration CDs increased in the solution. Samples treated with $4 \%$ and $6 \% \beta$-Cyclodextrin showed best results with visible increase in hydroxyl $(\mathrm{OH})$ stretch region of the spectrum indicating efficient attachment of $\beta$-Cyclodextrin to the fabrics.

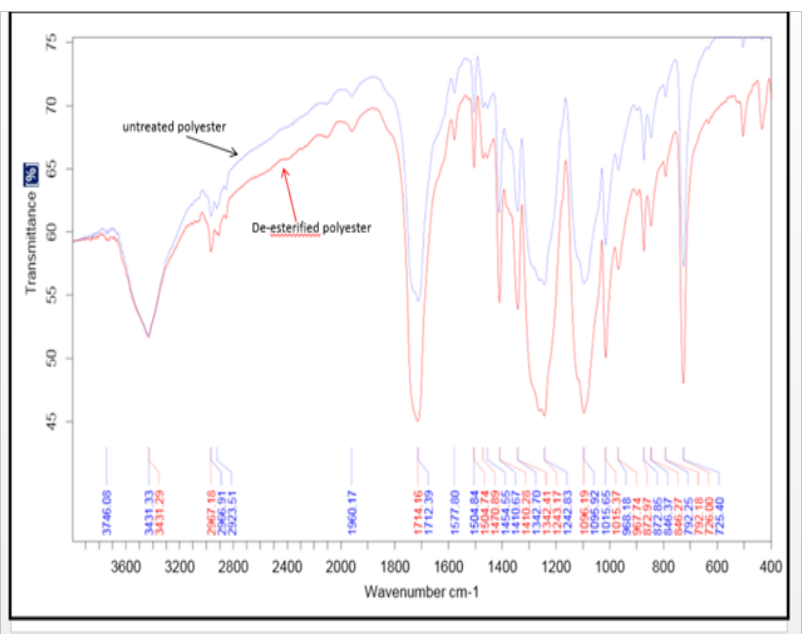

Figure 3 Shows the FTIR Spectra of before and after de-esterified polyester.

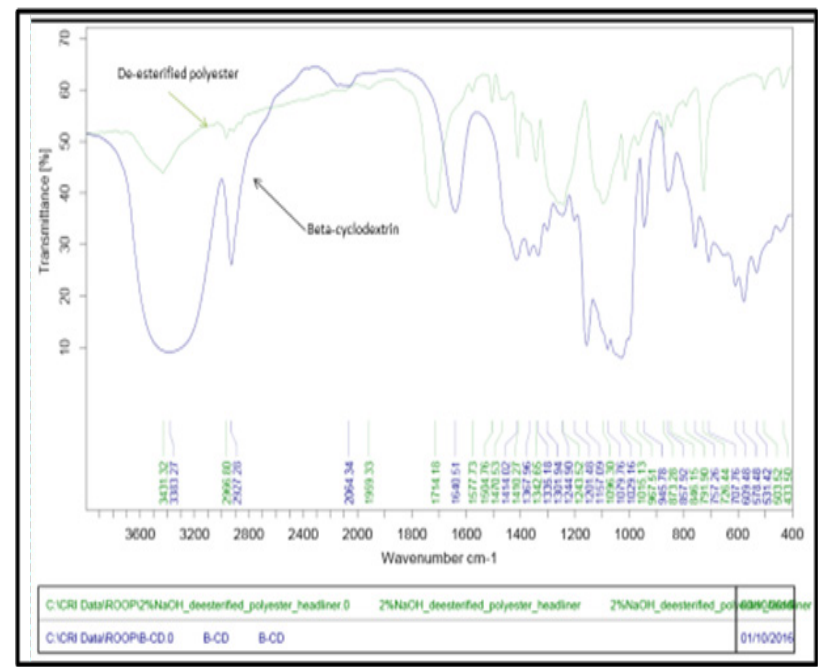

Figure 4 Illustrates the FTIR Spectra of De-esterified Polyester and $\beta$-Cyclodextrin.

\section{Phenolphthalein test for the detection of $\beta$-Cyclodex- trin}

$1 \%$ phenolphthalein solution is made in $50 \%$ ethanol solution in an alkaline medium. Two drops of that solution is applied to 
fabric samples having no $\beta$-Cyclodextrin, $2 \% \beta$-Cyclodextrin, $4 \%$ $\beta$-Cyclodextrin and $6 \% \beta$-Cyclodextrin. Pink color is retained in the no $\beta$-Cyclodextrin treated sample and $2 \% \beta$-Cyclodextrin treated sample. The samples treated with $4 \%$ and $6 \% \beta$-Cyclodextrin show no pink color as shown in Figure 6. This accounts for successful attachment of $\beta$-Cyclodextrin to the fabric substrate since $\beta$-Cyclodextrin discolors the color of pink alkaline phenolphthalein.

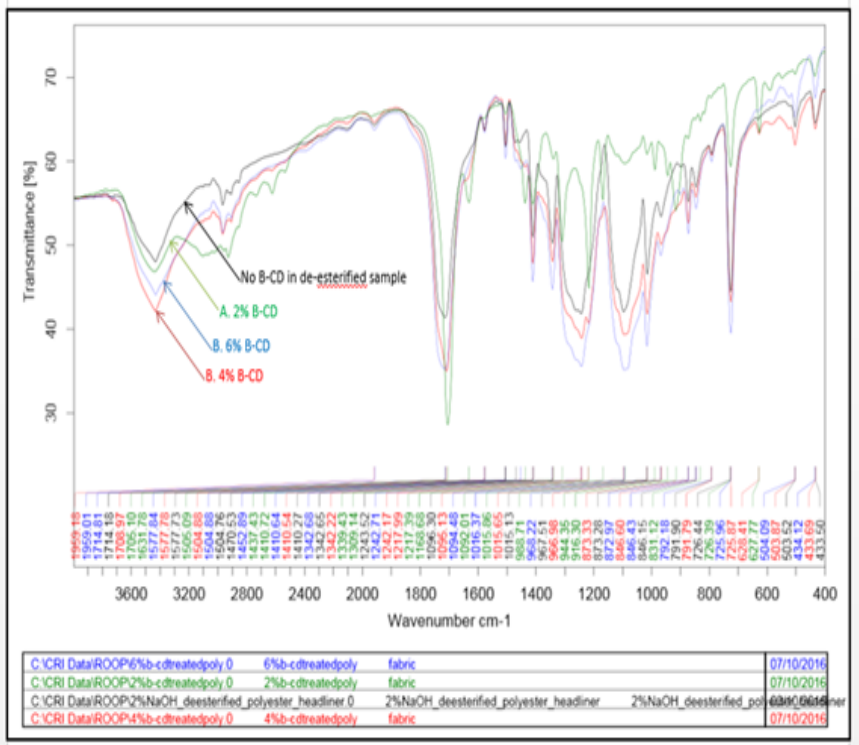

Figure 5 Shows the FTIR Spectra of de-esterified PET fabric treated with varying amounts of $\beta$-Cyclodextrin.

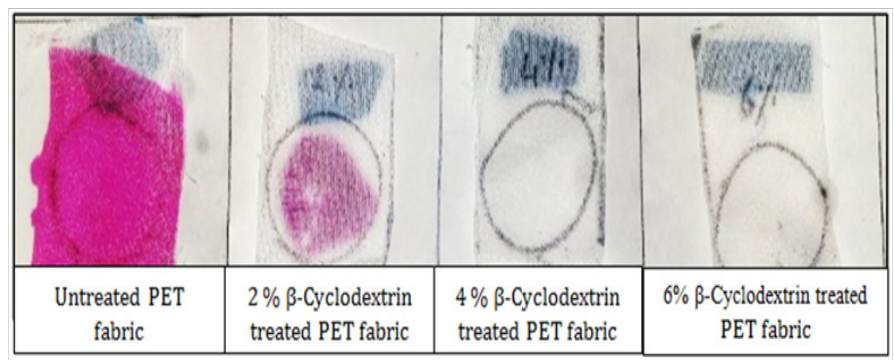

Figure 6 Shows the stain on PET fabric because of the application of phenolphthalein and gradual disappearance of stain due to the attachment of $\beta$-Cyclodextrin on the fabric.

\section{Subjective analysis of fragrance}

A group of 5 judges estimated the fragrance from the fabric weekly and graded the intensity on the scale of 0 to 5 , with 0 being no fragrance at all and 5 being the most intense fragrance. It was observed from this subjective analysis that the intensity of the fragrance decreased with increase of time, though the fragrance was retained in the fabric for about 4 weeks. With the optimization of the fragrance concentration and application technique this time period can be increased as found in a previous study. ${ }^{6}$ The fabric can be resprayed after certain time interval.

\section{Conclusion}

Polyester fabric de-esterified with $2 \% \mathrm{NaOH}$ and treated with $4 \%$ $\beta$-Cyclodextrin is proved to have formed a successful active agent releasing polyester fabric system. The fragrance retention of the treated fabric has to be further estimated by more comprehensive methods. More effective fragrance solution concentration and application technique to improve longevity is to be further investigated.

\section{Acknowledgements}

None.

\section{Conflict of interest}

There is no conflict of interest.

\section{References}

1. Brown D. Aromatherapy. Lincolnwood (Chicago): NTC Publishing Group; 1996. p. 51-60.

2. Hu J, Xiao Z, Ma SS, et al. Comparison of compounded fragrance and chitosan Nano particle loaded with fragrance applied in cotton fabrics. Textile Research J. 2011;81(19):2056-2064.

3. Wang CX, Chen SL. Surface treatment of cotton using $\beta$-Cyclodextrins sol-gel method. Applied Surface Science. 2006;252(18):6348-6352.

4. Martin GN. Olfactory Remediation: Current Evidence and Possible Applications. Soc Sci Med. 1996;43(1):63-70.

5. Huang L, Allen E, Tonelli A. Inclusion compounds formed between Cyclodextrins and nylon 6. Polymer. 1999;40(11):3211-3221.

6. Ghosh S, Chipot N. Embedding aromatherapy essential oils into textile fabric using Cyclodextrin Inclusion compound. Indian J Fibre \& Textile Research. 2015;40(2):140-143. 